\title{
A Comprehensive Evaluation and Analysis of Routing Algorithms for Wireless Sensor Networks
}

\author{
Arunkumar.A ${ }^{1}$, Dr.S.Prema ${ }^{2}$ \\ M. Phil Research Scholar, Department of Computer Science (PG), \\ K. S. Rangasamy College of Arts and Science (Autonomous), Tiruchengode, Tamilnadu, India ${ }^{1}$ \\ Associate Professor, Department of Computer Science (PG), \\ K. S. Rangasamy College of Arts and Science (Autonomous), Tiruchengode, Tamilnadu, India ${ }^{2}$
}

\begin{abstract}
A wireless sensor network is the prominent technology, which seeks lots of attention in the state of the art technology. It has its wide range of utilization aspects in almost all domains. The major challenge of the WSN seems to be the energy utilization and as a consequence the decline of the lifetime of the sensor nodes. This research addresses the problem of the energy drain in the sensor nodes lifetime, which as a result it ends up in the nodes death. This research proposes an algorithm to cope up with the energy utilization of the sensor nodes. Wireless sensor networks are deployed widely in sensitive applications like health care, surveillance and e-commerce domains. Compared with communication protocols, in terms of energy indulgence, ease of formation, and system epoch/quality of the network. Providing such a low-energy, ad hoc, distributed protocol will help pave the way for imminent micro sensor networks. It is evident from the MATLAB simulation that the proposed system works well efficient to cope up with energy efficient and novel clustering. In this research, novel constellation based wsn clustering protocol is proposed for the wireless sensor networks. It improves the efficiency of the energy consumption along with the improvement of the node lifetime values. The proposed strategy helps in improving the dynamic updating of the routing table, which may overcome the problem of the dead node sensing and cluster head value updating.
\end{abstract}

Keywords: Wireless Sensor Network, Routing Algorithm, MATLAB

\section{INTRODUCTION}

A computer network is a group of computer systems and other computing hardware devices that are linked together through the communication channels to ease of communication and resource-sharing and file sharing among a wide range of users. Networks are commonly categorized based on their characteristics. A Wireless sensor network can be defined as a network of devices that can communicate the information gathered from a monitored field through the wireless links. The data is forward through multiple nodes, and with a gateway, the data is connected to other networks like wireless Ethernet. Commonly monitored parameters and temperature, pressure, humidity, wind direction and speed, illumination intensity, vibration intensity, sound intensity,, chemical, power-line voltage, concentrations, pollutant levels and vital body functions.

\section{LITERATURE REVIEW}

Radio Sleep Mode Raja Jurdak et.al [1]: Energy efficiency is a middle challenge in sensor networks, and the radio is a major contributor to overall energy node utilization. Current energy efficient for MAC protocols used for sensor networks use a set low power radio mode for putting the radio to sleep. This paper proposes adaptive radio low-power sleep mode based on current traffic conditions inside the network. It first introduces a comprehensive node energy model, which includes energy components for radio switching, transmission, response, listening, and sleeping, as well as the often disregarded microcontroller energy component for determining the optimal sleep mode along with MAC protocols to use for specified traffic scenarios. This paper has proposed adaptive radio sleep modes as an energy optimization system for wireless sensor networks. Mobile Data Gathering Miao Zhao et.al [2] : Recent advances have shown a great potential of mobile data gathering in wireless sensor networks, where one or more mobile collectors are employed to collect the data from sensors using short-range of communications. Among several of data gathering approaches, one typical scheme is called anchor-based mobile data gathering. In this paper, we focus on such a data gathering method and provide distributed algorithms to achieve its optimal performance. We consider two different cases depending on whether the mobile collector has fixed or variable stay time at each anchor point. Finally, we provide extensive numerical results to demonstrate the usage and efficiency of the proposed algorithms and set off our theoretical analysis. 
Routing Design in Wireless Sensor Networks Peng Guo, Xuefeng Liu et.al [3]: In many domain-specific monitoring applications of wireless sensor networks (WSNs), such as structural health monitoring, volcano tomography, and machine diagnosis, the raw data in WSNs are required to be listlessly gathered to the sink, where a focused centralized algorithm is then executed to extract some global features or model parameters. we present a series of novel routing schemes customized for different cases of computation results. The work in this paper can also serve as a guideline for distributed computing of big data, where the data spreads in a large network.

Ant Colony Optimization Yongjun Sun et.al [4]: In order to find the optimal path of data transmission in the WSNs, a new routing algorithm based on ant colony algorithm is proposed. Using the improved heuristic function and considering the node communication transmission distance, transmission direction, and residual energy, an optimal path from the source node to the destination node can be found Simulation results show that new ant algorithm can effectively save the energy of nodes and prolong the network lifetime. The simulation results indicate with the purpose of by comparing with EEABR, Leach-Ant and OARA, the method proposed in the paper obviously minimizes the average energy consumption and extends the life cycle of the wireless sensor network.

Network-on-Chip Liang Wang et.al [5]: Technology scaling leads to the reliability issue since a primary concern in Network-on-Chip (NoC) design. We observe that due to routing algorithm routers age much faster than others which become a bottleneck for NoC life time. In this paper, network on chip paper lifetime is modeled as a resource consumed over time. A metric lifetime budget is associated with each router, indicating the maximum allowed workload for current period. Since the heterogeneity in router lifetime reliability has strong correlation with the routing algorithm, we define a problem to optimize the lifetime by routing packets along the path with maximum lifetime budgets.

Table I Summary of Literature Review

\begin{tabular}{|c|c|c|c|c|c|}
\hline $\begin{array}{l}\text { S. } \\
\text { No }\end{array}$ & itle & $\begin{array}{l}\text { Author } \\
\text { Publisher } \\
\text { and Year }\end{array}$ & Working Platform & bjective & Future Scope \\
\hline 1 & $\begin{array}{l}\text { Radio Sleep Mode } \\
\text { Optimization in } \\
\text { Wireless Sensor } \\
\text { Networks }\end{array}$ & $\begin{array}{l}\text { Raja Jurdak, } \\
\text { Member, } \\
\text { IEEE, } \\
{[2010]}\end{array}$ & $\begin{array}{ll}\text { Wireless } & \text { Sensor } \\
\text { Networks } & \end{array}$ & $\begin{array}{lr}\text { radio is a } & \text { major } \\
\text { contributor } & \text { to } \\
\text { overall } & \text { energy } \\
\text { node consumption }\end{array}$ & $\begin{array}{l}\text { This paper has proposed } \\
\text { adaptive radio } \\
\text { modes as an energy } \\
\text { optimization } \\
\text { systemfor } \\
\text { sensor networks }\end{array}$ \\
\hline 2 & \begin{tabular}{lr}
\multicolumn{2}{l}{ Optimization-Based } \\
Distributed & \\
Algorithms & for \\
Mobile & Data \\
Gathering & in \\
Wireless & Sensor \\
Networks &
\end{tabular} & $\begin{array}{l}\text { Miao Zhao, } \\
\text { Member, } \\
\text { IEEE, } \\
{[2012]}\end{array}$ & $\begin{array}{l}\text { Mobile } \\
\text { Gathering }\end{array}$ & $\begin{array}{l}\text { Among a variety of } \\
\text { data gathering } \\
\text { approaches, one } \\
\text { typical scheme is } \\
\text { called anchor- } \\
\text { based mobile data } \\
\text { gathering }\end{array}$ & 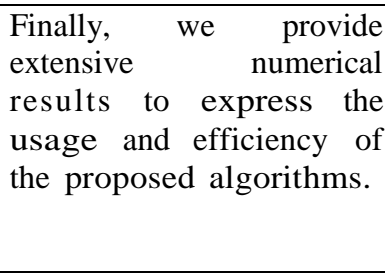 \\
\hline 3 & $\begin{array}{l}\text { Lossless In-Network } \\
\text { Processing and Its } \\
\text { Routing } \\
\text { Design in Wireless } \\
\text { Sensor Networks }\end{array}$ & $\begin{array}{l}\text { Peng Guo, } \\
\text { IEEE } \\
{[2017]}\end{array}$ & $\begin{array}{l}\text { Wireless } \\
\text { network } \\
\text { in-network } \\
\text { processing, matrix } \\
\text { computation, routing } \\
\text { scheme. }\end{array}$ & $\begin{array}{lr}\text { many } & \text { domain- } \\
\text { specific monitoring } & \text { mof } \\
\text { applications } & \text { of } \\
\text { wireless } & \text { sensor } \\
\text { networks } & \text { (WSNs), } \\
\text { such as structural } \\
\text { health monitoring, }\end{array}$ & $\begin{array}{l}\text { The work in this paper } \\
\text { can also serve as a } \\
\text { guideline for distributed } \\
\text { computing of big data, } \\
\text { where the data spreads in a } \\
\text { large network. }\end{array}$ \\
\hline 4 & $\begin{array}{l}\text { An Improved Routing } \\
\text { Algorithm Based on } \\
\text { Ant Colony } \\
\text { Optimization in } \\
\text { Wireless Sensor } \\
\text { Networks }\end{array}$ & $\begin{array}{l}\text { Yongjun Sun, } \\
\text { IEEE, [2017] }\end{array}$ & $\begin{array}{l}\text { Wireless rensor } \\
\text { networks, routing } \\
\text { algorithms, ant } \\
\text { colony optimazation } \\
\text { energy consumption, } \\
\text { network lifetime }\end{array}$ & $\begin{array}{l}\text { Data routing in } \\
\text { energy } \\
\text { constrained wireless } \\
\text { sensor networks } \\
\text { (WSNs) is one of } \\
\text { the key points. }\end{array}$ & $\begin{array}{l}\text { Proposed in the paper } \\
\text { obviously minimizes the } \\
\text { average and energy } \\
\text { consumption and extends } \\
\text { the life cycle of the } \\
\text { wireless sensor network. }\end{array}$ \\
\hline 5 & $\begin{array}{l}\text { Adaptive Routing } \\
\text { Algorithms for } \\
\text { Lifetime } \\
\text { Reliability } \\
\text { Optimization in } \\
\text { Network-on-Chip }\end{array}$ & $\begin{array}{l}\text { Liang Wang, } \\
\text { IEEE, } \\
{[2016]}\end{array}$ & $\begin{array}{l}\text { Routing algorithm, } \\
\text { network-on-chip, } \\
\text { lifetime reliability, } \\
\text { dynamic } \\
\text { programming }\end{array}$ & $\begin{array}{l}\text { Technology scaling } \\
\text { leads to the } \\
\text { reliability issue as a } \\
\text { primary distress in } \\
\text { Network-on-Chip } \\
\text { (NoC) design. }\end{array}$ & $\begin{array}{l}\text { In this paper, we } \\
\text { propose dynamic } \\
\text { programming-based } \\
\text { lifetime aware routing } \\
\text { algorithms for NoC } \\
\text { reliability management }\end{array}$ \\
\hline
\end{tabular}




\section{NOVEL CONSTELLATION WIRELESS SENSOR NETWORKS (NCWSN) ROUTING}

\section{Wireless Sensor Networks}

Wireless sensor networks are deployed widely in sensitive applications like health care, surveillance and e-commerce domains. Like other sensors, WSN needs routing and typically, the routing will consider the number of nodes used, environment, event and movement of sensors.

Routing in WSN focus on the following aspects:

1. Environment based movement monitoring

2. Traffic observation

3. Sensor signal

4. Alive and dead nodes ratio

\section{WSN Topology And Routing Perspectives}

Different type of topologies is adopted in WSN, which may consider the following types of routing to accomplish the task of the communication.
- Hierarchical
- Location based
- Direct contact

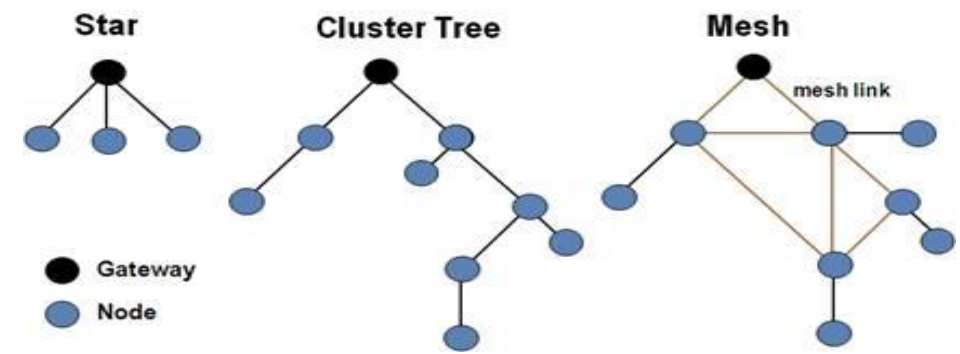

Figure 1: Common WSN Network Topologies

The sensors involved in the WSN are tiny nodes with sense ability, compute ability and communication ability. An essential and most significant design challenge is the energy efficient awareness of the sensor nodes to retain the life in order to accomplish the task it has been made for.

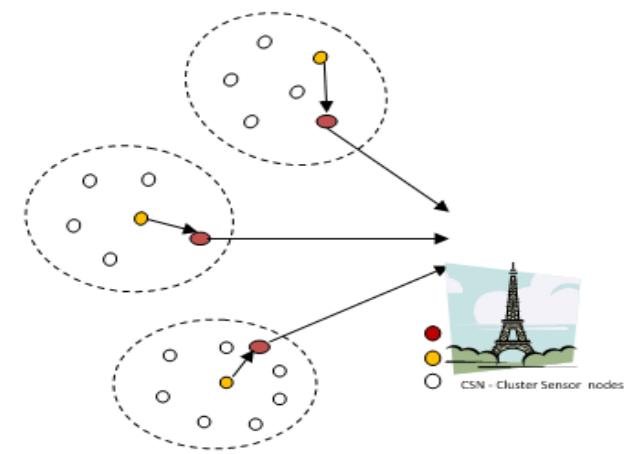

Figure 2: Routing of WSN in cluster manner

\section{NCWSN - PROPOSED WORK}

The proposed work is creating a routing algorithm for the WSN which address the address the problem of the routing energy drain and packet loss.

$$
\begin{aligned}
\text { NCWSN }= & \frac{R S_{x}}{\sum \text { Links connectiong to } x\left(R Q_{y}+\alpha\right)} \\
& \quad-\frac{R S_{x}}{\sum \text { Links connectiong to } x\left(\operatorname{Dist}(x)^{2}+\beta+\alpha\right)}
\end{aligned}
$$

Now the simple calculation for the empirical values for figure 4.2 is as follows: 


$$
\begin{gathered}
N C W S N_{C H(A)}=\frac{2.1 j}{1.2^{2}+1.2^{2}+1.1^{2}+0.4^{2}}=0.4941 \\
N C W S N_{C H(B)}=\frac{2.3 j}{1.2^{2}+1.2^{2}+0.5^{2}+1.4^{2}}=0.4518 \\
N C W S N \vdots_{C H(C)}=\frac{1 j}{0.8^{2}+0.4^{2}}=1.25
\end{gathered}
$$

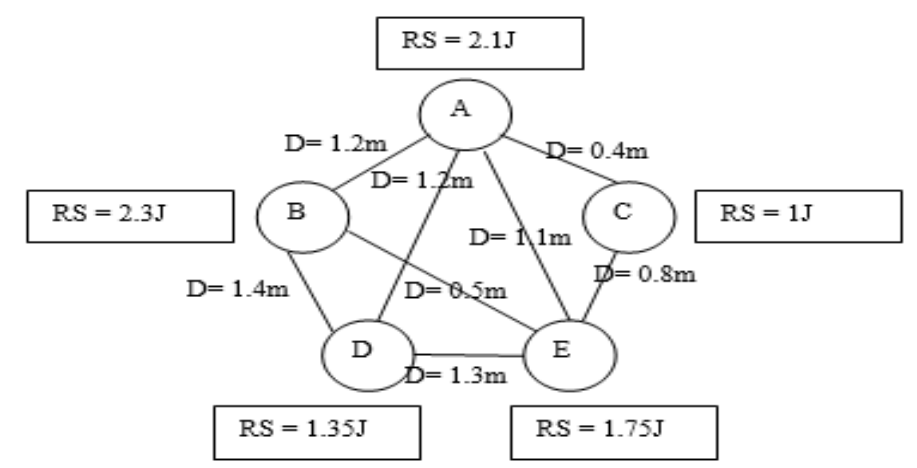

Figure 3: NCWSN scenario empirical value calculations

\section{NCWSN IMPLEMENTATION}

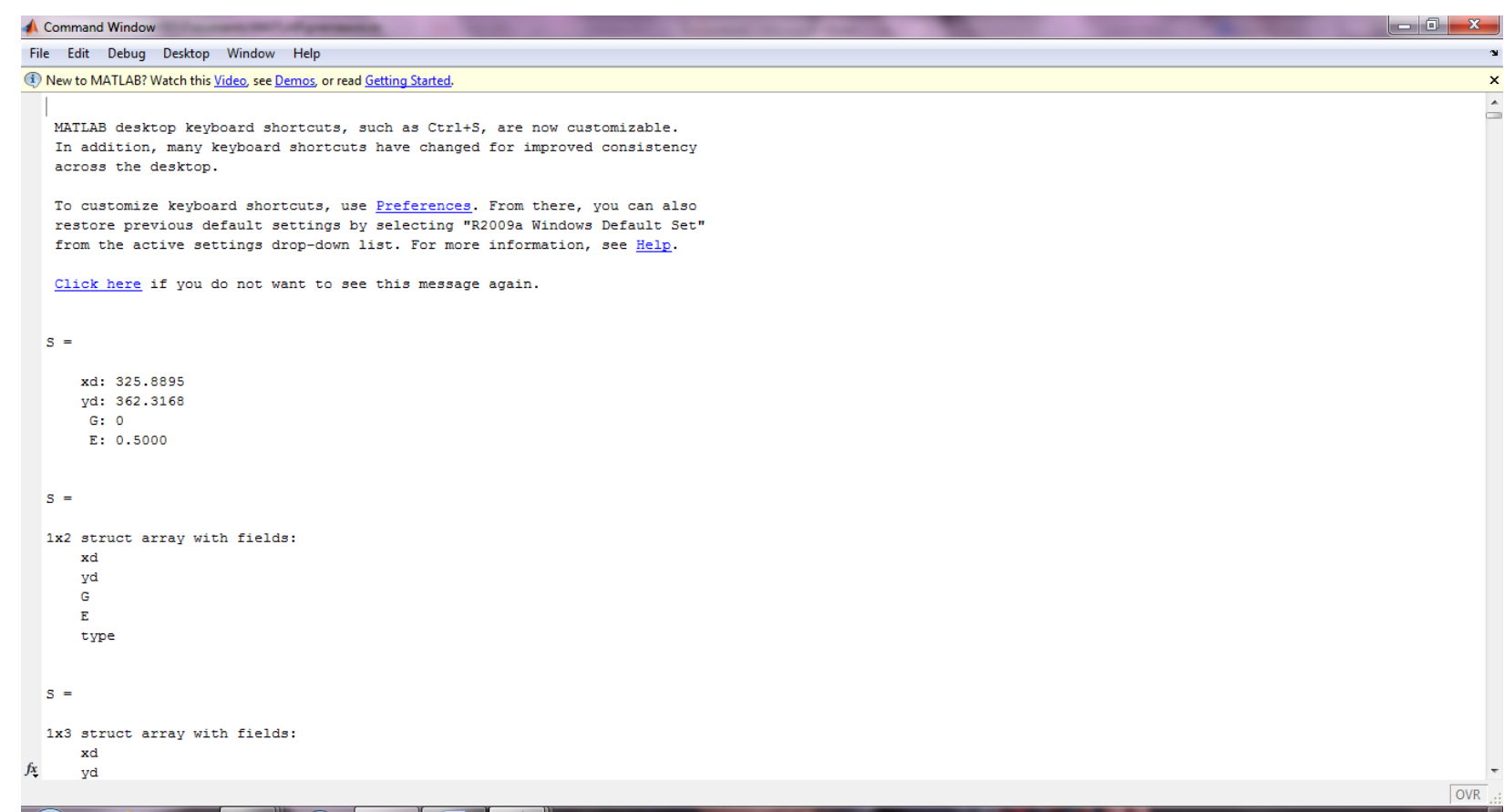

Figure4: Ncwsn Implementation

\section{IMPLEMENTATION OF THE PROPOSED WORK}

\section{Results And Discussion}

It is evident from the MATLAB simulation that the proposed system works well efficient to cope up with energy efficient and novel clustering. Compared with communication protocols, in terms of energy indulgence, ease of formation, and system epoch/quality of the network. Providing such a low-energy, ad hoc, distributed protocol will help pave the way for imminent micro sensor networks. Help pave the way for imminent micro sensor networks. 
Vol. 7, Issue 11, November 2018

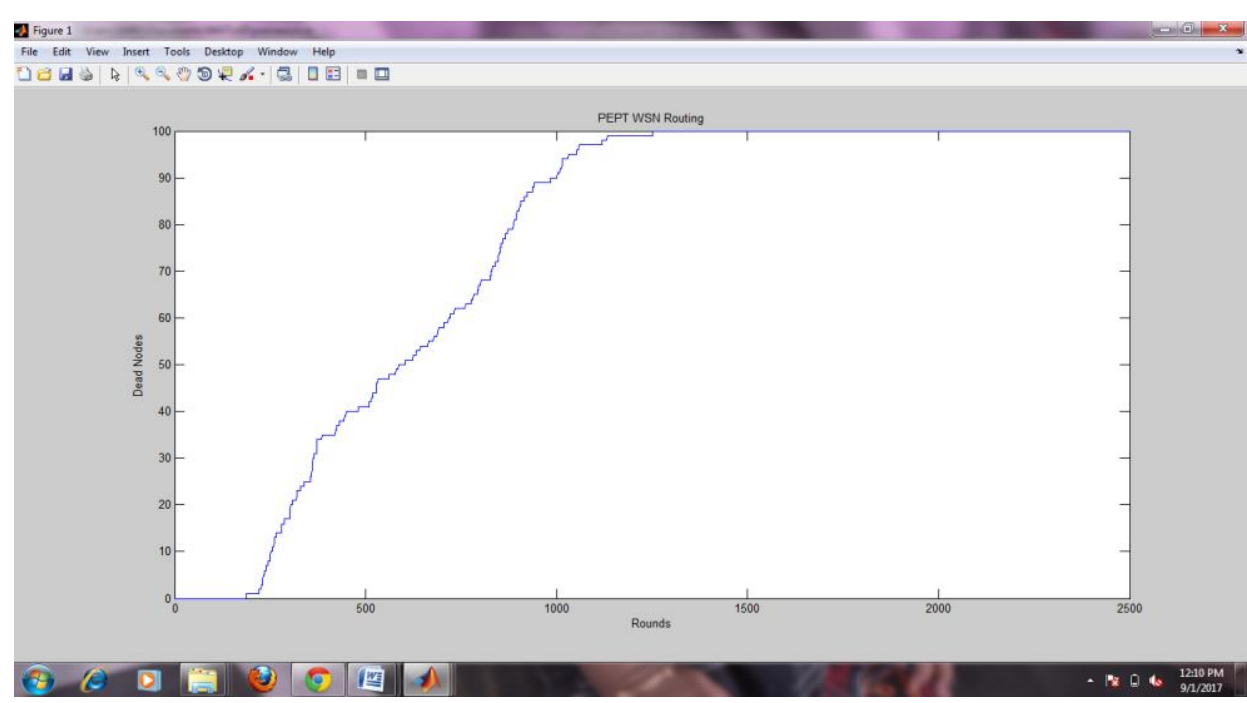

Figure 5: Nodes Alive Ratio

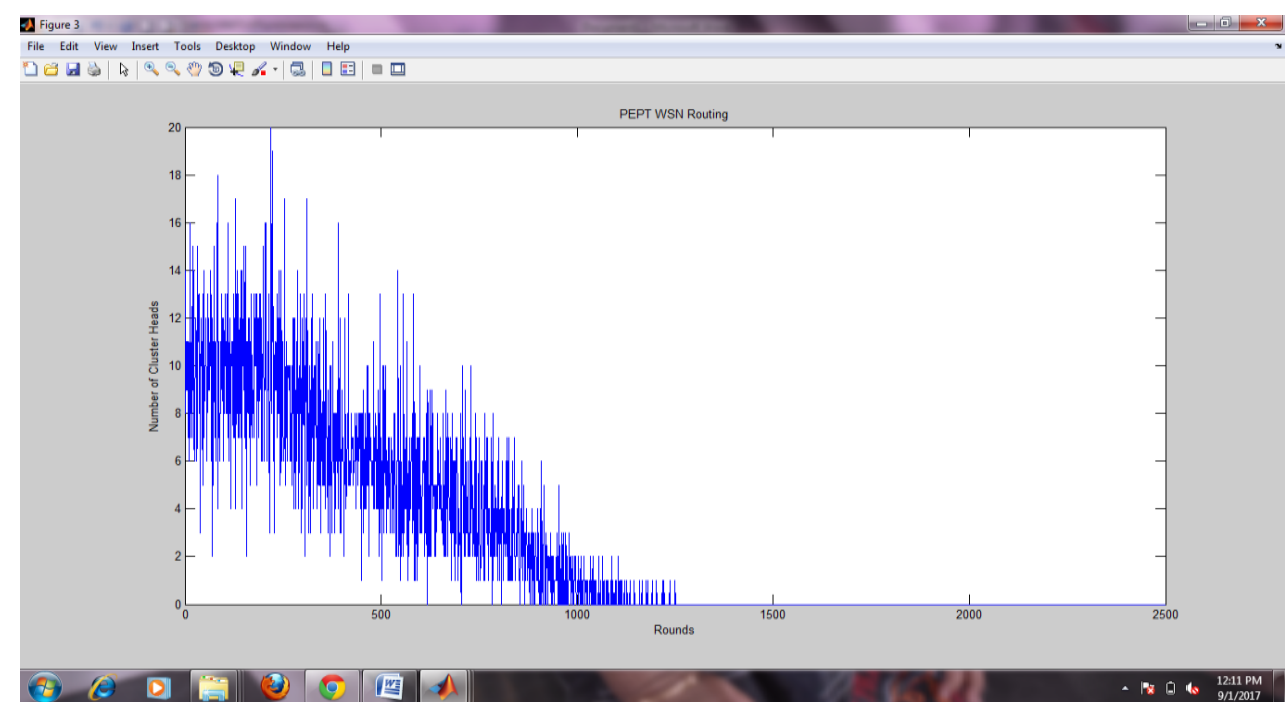

Figure 6: Representative Ratio among the Nodes

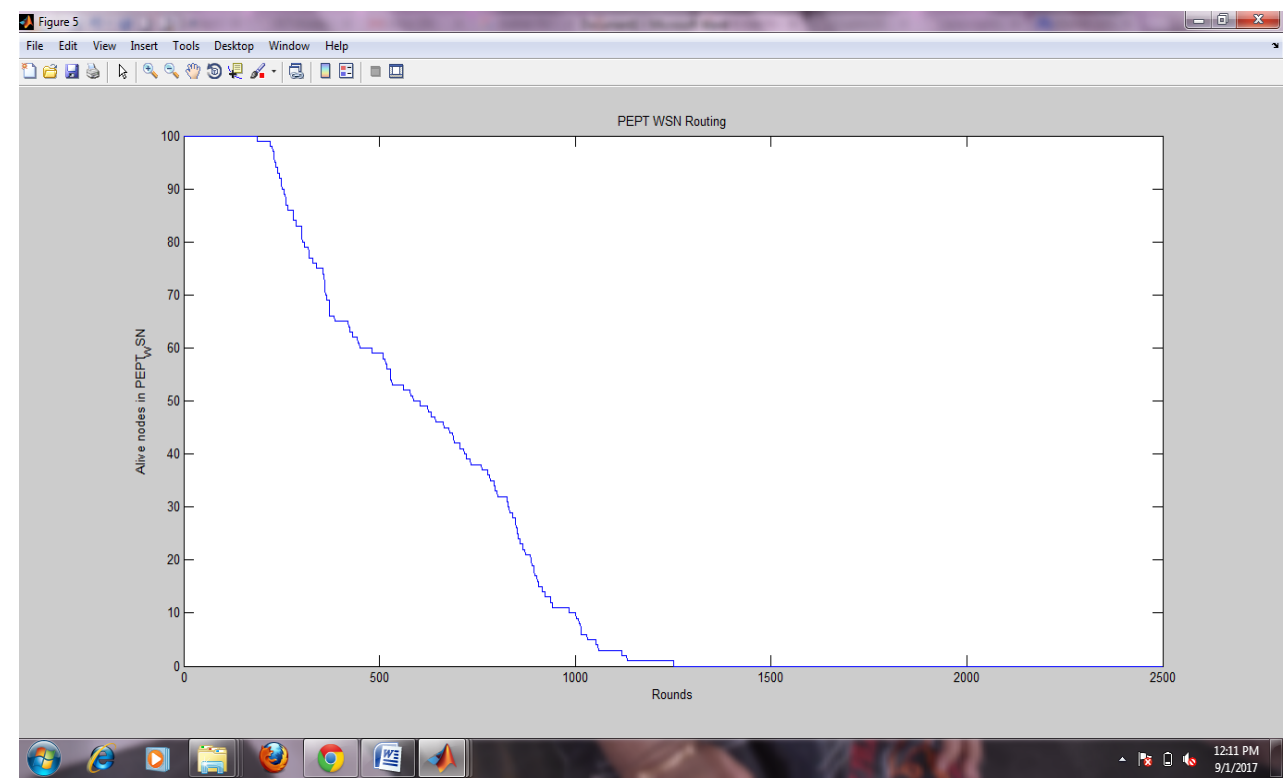

Figure 7: Alive Nodes Ratio 
Vol. 7, Issue 11, November 2018

\section{SUMMARY}

In this research, novel constellation based wan clustering protocol is proposed for the wireless sensor networks. It improves the efficiency of the energy consumption along with the improvement of the node lifetime values. The proposed strategy helps in improving the dynamic updating of the routing table, which may overcome the problem of the dead node sensing and cluster head value updating.

\section{CONCLUSION}

Compared with communication protocols, in terms of energy indulgence, ease of formation, and system epoch/quality of the network. Providing such a low-energy, ad hoc, distributed protocol will help pave the way for imminent micro sensor networks. It is evident from the MATLAB simulation that the proposed system works well efficient to cope up with energy efficient and novel clustering. In this research, novel constellation based WSN clustering protocol is proposed for the wireless sensor networks. It improves the efficiency of the energy consumption along with the improvement of the node lifetime values. The proposed strategy helps in improving the dynamic updating of the routing table, which may overcome the problem of the dead node sensing and cluster head value updating. It is evident from the simulation that the proposed system works well efficient to cope up with energy efficient and novel clustering. It is concluded that the proposed system performs well in terms of the metrics considered

\section{CONTRIBUTIONS TO THE KNOWLEDGE}

This research addresses the problem of the energy drain in the sensor nodes lifetime, which as a result it ends up in the nodes death. This research proposes an algorithm to cope up with the energy utilization of the sensor nodes. Wireless sensor networks are deployed widely in sensitive applications like health care, surveillance and e-commerce domains.

\section{FURTHER ENHANCEMENT}

Concentrating on the dynamic update of the route table and route selection can improve the performance of the WSN routing. And also the support of heterogeneous type of the sensor nodes can yield the further enhancement in the performance of the WSN routing. This could be the further enhancement for this research.

\section{REFERENCES}

[1]. Ahmed E.A.A. Abdulla. "Hymn: A Novel Hybrid Multi-Hop Routing Algorithm To Improve The Longevity Of Wsns" IEEE Transactions On Wireless Communications, Vol. 11, No. 7, July 2012.

[2]. Miao Zhao,"Optimization-Based Distributed Algorithms For Mobile Data Gathering In Wireless Sensor Networks" IEEE Transactions On Mobile Computing, Vol. 11, No. 10, October 2012.

[3]. Peng Guo, "Lossless In-Network Processing and Its Routing Design In Wireless Sensor Networks" IEEE Transactions On Wireless Communications, Vol. 16, No. 10, October 2017.

[4]. Yongjun Sun, "An Improved Routing Algorithm Based On Ant Colony Optimization in Wireless Sensor Networks" IEEE Communications Letters, Vol. 21, No. 6, June 2017.

[5]. Liang Wang, Xiaohang Wang "Adaptive Routing Algorithms For Lifetime Reliability Optimization In Network-On-Chip" IEEE Transactions On Computers, Vol. 65, No. 9, September 2016.

\section{BIOGRAPHIES}

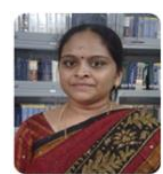

Dr. S.Prema, currently working as an Associate Professor in K.S.Rangasamy College of Arts \& Science has received Ph.D., from the Bharathiar University in 2015. She has been involved in the teaching for the past 13 years. She secured the $1^{\text {st }}$ Rank in B.Sc under Periyar University, Salem. She has totally 52 publications and one of her research paper entitled "An NLP based Approach for Facilitating Efficient Web Search Results using BSDS"received the best paper award. Her papers are cited at various publications (IEEE Xplore, Elsevier, Springer and International Conference Proceedings). She has h-index value: 5, i10 index: 3, Citations: 125 and her profile is listed in Marquis Who is Who in World, International Biography Center London, UK, 2011. She has been awarded "Innovative Research \& Dedicated Women Academician Award" at International Awards \& Honors Convocation 2018 Conducted by The Society of Innovative Educationalist \& Scientific Research Professional, Malaysia. She has produced 5 M.Phil scholars and currently guiding 4 M.Phil and 1 Ph.D Scholars for doing their research. She has been involved in generating funds for R\&D.

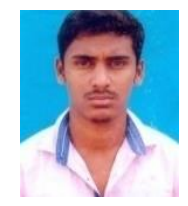

Mr. Arunkumar .A is pursuing M.Phil (Computer Science) in K.S.Rangasamy College of Arts and Science (Autonomous), Tamilnadu, India. He has completed M.SC (CS) degree under Periyar University, in 2016. He has attended 3 workshops, 1 seminar related to Network and presented a paper related to his research work in an International conference. His areas of interest are Network and Data Mining. 